\title{
CARACTERÍSTICAS CLÍNICO-EPIDEMIOLÓGICAS DE LA INFECCIÓN RESPIRATORIA AGUDA (IRA) POR VIRUS SINCITIAL RESPIRATORIO (VSR) EN NIÑOS MENORES DE DOS AÑOS ADMITIDOS EN EL HOSPITAL INFANTIL DR. ROBERT REID CABRAL (HIRRC), ENERO-DICIEMBRE 2006
}

\section{Clinical-epidemiological characteristics of Acute Respiratory Infection (IRA) by Respira- tory Syncitial Virus (RSV) In children under two years admitted in Hospital Infantil Dr. Robert Reid Cabral (HIRRC) January-December 2006}

\section{Virgen Gómez Alba*, Jesús Feris-Iglesias *, Angélica Florén**, Jacqueline Sánchez*, Josefina Fernández*}

Recibido: 20/3/2018 Aprobado: 22/4/2018

Cómo citar: Gómez-Alba V, Feris-Iglesias J, Florén A, Sánchez J, Fernández J, Características clínico-epidemiológicas de la infección respiratoria aguda (ira) por Virus Sincitial Respiratorio (VSR) en niños menores de dos años: admitidos en el hospital Infantil Dr. Robert Reid Cabral (HIRRC), enero-diciembre 2006 Ciencia y Salud [Internet]. 2018 mayo 1; [citado 2018 agosto 15]; 2(2):[aprox. 0 p.]. Disponible en: https://revistas.intec.edu.do/index.php/ cisa/article/view/1182

\section{Resumen}

Antecedentes: el VSR es el principal responsable de bronquiolitis y neumonía intersticial en los primeros 2 ańos de vida y se asocia a complicaciones bacterianas e incremento de hospitalizaciones por infecciones respiratorias.

Los casos ocurren usualmente en otońo e invierno. En República Dominicana se desconoce el comportamiento clínico y epidemiológico de la IRA causada por VRS.

\footnotetext{
*Departamento Enfermedades Infecciosas, Hospital Infantil Dr. Robert Reid Cabral, Santo Domingo, República Dominicana.

**Jackson Memorial Hospital, Miami, Fl, EE. UU.

E-correo: virgen.gomez@yahoo.es

Hospital Dr. Robert Reid Cabral. jesusferis@yahoo.es

florenangelica@aol.com

jackeline1954@yahoo.es
}

Hospital Dr. Robert Reid Cabral. infectologia01@yahoo.es
Objetivo: determinar las características clínicas y epidemiológicas de los casos de IRA por VRS en niños menores de 2 años durante el período de estudio.

Métodos: estudio prospectivo, descriptivo y observacional donde se estudiaron todos los niños de $0 \mathrm{a}$ 24 meses ingresados con diagnóstico de bronquiolitis durante el período de estudio y radiografías de tórax reportadas como bronquiolitis o neumonía alveolo-intersticial, que fueron confirmadas por la prueba Binax Now VRS.

Resultados: de 114 niños de 0-24 meses de edad y de ambos sexos, admitidos con diagnóstico de bronquiolitis en el HIRRC durante el período de estudio, en 54 casos (48\%) se confirmó el VRS a través de la prueba Binax Now VRS, $62 \%$ correspondió al sexo masculino, el $76 \%$ a los menores de 4 meses; tos y dificultad respiratoria fueron las manifestaciones clínicas más frecuentes, con 52 casos (94.5\%), seguidas por sibilancias, con 46 (83.6\%) 
y retracción subcostal, con 41 (74.5\%); la radiografía de tórax reportó bronquiolitis en 36 casos (66.7\%), neumonía alveolo-intersticial en 16 casos (29.6\%), neumonía más neumotórax y bronquiolitis más atelectasia en 1 caso (1.8\%), respectivamente. En el $76.3 \%$ la estadía fue menor de 4 días. La totalidad de los casos se presentaron en los meses de octubre (19 casos), noviembre (22 casos), diciembre ( 12 casos) y enero ( 1 caso); el $87 \%$ presentó anemia, hallazgo no reportado en otros estudios.

Conclusión: VSR tiene comportamiento estacional; es el responsable de la mayoría (48\%) de los casos de bronquiolitis y neumonía intersticial en lactantes menores; tos y dificultad respiratoria fueron las manifestaciones clínicas más frecuentes; la anemia estuvo presente en el $87 \%$ de los casos.

Palabras clave: Virus sincitial respiratorio; bronquiolitis; neumonía intersticial; comportamiento estacional; anemia.

\section{Summary}

Background: RSV is the main cause of bronchiolitis and interstitial pneumonia in the first 2 years of life and is associated with bacterial complications and increased hospitalizations for respiratory infections.

The cases usually occur in autumn and winter. In the Dominican Republic the clinical and epidemiological compartments of the ARF caused by RSV are unknown.

Objectives: To determine the clinical and epidemiological characteristics of cases of ARI for RSV in children under 2 years old during the study period.
Methods: Prospective, descriptive and observational study in which all children from 0 to 24 months admitted with a diagnosis of bronchiolitis during the study period and chest X-rays reported as bronchiolitis or alveolar-interstitial pneumonia were studied and confirmed by the Binax Now ${ }^{\bullet}$ RS test.

Results: Of 114 children between 0-24 months of age and of both sexes, who were admitted with a diagnosis of bronchiolitis in the HIRRC during the study period, 54 cases (48\%) confirmed the RSV through the Binax Now test. VRS, 62 percent corresponded to males, 76 percent to children under 4 months; cough and respiratory distress were the most frequent clinical manifestations with 52 cases $(94.5 \%)$, followed by wheezing (46) (83.6\%) and subcostal retraction (41) (74.5\%), chest radiography reported bronchiolitis in 36 cases $(66.7 \%)$ pneumonia alveolus -interstitial in 16 cases $(29.6 \%)$, pneumonia plus pneumothorax and bronchiolitis plus atelectasis in 1 case $(1.8 \%)$ respectively. In $76.3 \%$ the stay was less than 4 days. All cases were presented in the months of October (19 cases), November (22 cases), December (12 cases) and January (1 case), and $87 \%$ presented anemia, a finding not reported in other studies.

Conclusion: RSV has seasonal behavior, is responsible for the majority (48\%) of cases of bronchiolitis and interstitial pneumonia in younger infants, cough and respiratory distress were the most frequent clinical manifestations, anemia was present in $87 \%$ of cases.

Keywords: Respiratory syncytial virus; bronchiolitis; interstitial pneumonia; seasonal behavior; anemia. 


\section{Introducción}

El VSR es la causa más frecuente de infecciones respiratorias bajas (IRABs), neumonías y bronquiolitis, en niños. Afecta a menores de 2 años, produciendo una enfermedad severa, que se asocia a complicaciones como Otitis Media Aguda (OMA), sinusitis, asma bronquial, con una alta tasa de morbimortalidad; aunque puede afectar a personas de cualquier edad ${ }^{1-8}$.

Existen dos grupos del VSR y varios subgrupos, los cuales pueden circular simultáneamente durante las epidemias invernales, y han sido detectados en todas las regiones del mundo. A diferencia de los virus de influenza y rinovirus, estos tienen menor variabilidad genética ${ }^{9-11}$.

El VSR suele producir brotes estacionales, comenzando a fines del otońo y persistiendo durante el invierno. Sin embargo, pueden surgir casos esporádicos durante todo el año. Circula de noviembre a marzo y afecta a más de los dos tercios de lactantes en el primer año de vida. A los dos años de edad el $95 \%$ de los niños han padecido la enfermedad. De estos, el 30\% desarrolla enfermedad de vías respiratorias bajas, el $1 \%$ amerita ingreso y cerca del $0,1 \%$ fallece ${ }^{12-14}$.

Aunque los menores de 2 años son los más afectados y desarrollan cuadro de mayor severidad, personas de la tercera edad pueden enfermar a causa de neumonitis y neumonía intersticial severa, conllevando a un incremento importante en las hospitalizaciones durante las epidemias ${ }^{1-3,15}$.

\section{Material y métodos}

Del $1 .^{\circ}$ de enero al 31 de diciembre de 2006 en el Hospital Infantil Dr. Robert Reid Cabral realizamos un estudio observacional, descriptivo, prospectivo y transversal que incluyó 114 niños de ambos sexos, con edades comprendidas entre 0 y 24 meses, con diagnóstico de bronquiolitis o neumonía intersticial, las que, confirmadas por radiografía de tórax, ameritaron ingreso. Sus padres o tutores aceptaron participar en el estudio y firmaron el formulario del consentimiento informado, con el objetivo de determinar VSR como agente etiológico.

A todos los niños se les realizó un hisopado nasofaríngeo y una prueba con el Test de Binax NOW VRS, ensayo inmunocromatográfico rápido que detecta la proteína de fusión del virus; prueba diseñada para facilitar el diagnóstico de infecciones por VSR en < 5 años,con una sensibilidad y especificidad del $93 \%{ }^{16}$.

Para el análisis estadístico se utilizaron los programas EPI-Info 6.04b, SPSS y CIA. Se calcularon las proporciones simples y sus intervalos de confianza al 95\%. Para comparación de variables nominales y numéricas se utilizaron el CHI cuadradoy la T de Student.

\section{Resultados}

Durante el período de estudio en el HIRRC se asistieron 99,751 pacientes; de estos, 66,895 correspondieron a consultas y 32,856 a emergencias; 23,810 casos $(23,8 \%)$ a IRA, de estos, 2,285 (9,6\%) fueron ingresados.

Del total de ingresos por IRA, 114 (4,98\%) cumplieron con los criterios de inclusión y se enrolaron en el estudio, se les realizó Rx de tórax, hemograma e hisopado nasofaríngeo para Binax NOW VRS, confirmándose VRS en 54 casos (48\%).

El $65 \%$ de los casos procedía de Santo Domingo, seguido de Haina y San José de Ocoa, con 9,3\% cada uno.

Durante todo el año se reportaron casos de bronquiolitis, excepto en junio y septiembre, confirmándose el VSR en 54 casos (48\%), ocurriendo estos en octubre, noviembre, diciembre y enero (ver gráfico 1). 
Hubo predominio del sexo masculino, con un 61\%; el grupo de edad de 0-2 meses fue el más afectado 40 casos (74\%), seguido por el de 3-5 meses, con 13 casos (24\%); no se presentó ningún caso en niños mayores de 7 meses, (IC 95\% 21 días-7 meses).

En cuanto a factores de riesgo, tener hermanos, otro miembro de la familia con tos, historia familiar de asma y hermanos que asisten a la escuela, fueron los factores asociados con mayor frecuencia; diferente a lo reportado en otros estudios donde la prematuridad, la no lactancia materna, el hacinamiento y el grado educativo de los padres se consideraron como los factores de riesgos predominantes (ver cuadro No. 1).

Por otra parte, tos, dificultad respiratoria, sibilancias y retracción supra-esternal fueron las manifestaciones clínicas más frecuentes (ver cuadro No. 2).

En el 87\% de casos se reportó anemia; no hubo diferencias importantes en cuanto a conteo de blancos, diferencial y plaquetas.

La radiografía de tórax confirmó datos de bronquiolitis en el $64.9 \%$ de los casos, neumonía intersticial en $31.5 \%$, neumonía más neumotórax y atelectasia en el $1.8 \%$, respectivamente.

En el $79.8 \%$, la estadía hospitalaria promedio fue de 4.1 días (IC 95\% 1-15). El 100\% evolucionó satisfactoriamente. No se reportaron secuelas respiratorias ni mortalidad.

\section{Discusión}

En 2005 unos 33,8 (IC 95\%: 19,3-46,2) millones de nuevos episodios de VRS asociados a infecciones respiratorias agudas bajas se produjeron en todo el mundo en menores de 5 años (22\%), y (3.4 2.8-4.3) millones de episodios estuvieron asociados aVRS grave requirieron ingreso hospitalario ${ }^{17}$.

Cada año el VSR suele producir brotes estacionales, comenzando a fines del otońo y persistiendo durante el invierno y se considera responsable de las epidemias de IRAB en esta estación. Sin embargo, pueden ocurrir casos esporádicos durante todo el año. En nuestro estudio se encontró que el 98.2\% de los casos ocurrieron en los meses octubre a diciembre, como sucede a nivel mundial (ver gráfico No. 1). ${ }^{18}$

Múltiples estudios confirman que el VSR es el responsable del 50\% de las IRABs virales en lactantes que ameritan ingreso, similar a lo confirmado en nuestro estudio (48 por ciento) de los casos ${ }^{18,19}$.

Se estima que durante el primer año de vida el $60 \%$ de los nińos han padecido la enfermedad y que a los dos años la mayoría ha presentado infección por VSR. En nuestro estudio todos los casos correspondieron a los menores de 7 meses y el grupo más afectado fueron los menores de 3 meses, con 45 casos $(89 \%)^{12-14}$.

La edad cronológica se considera como el predictor más importante de bronquiolitis grave, ya que estudios reportan que los dos tercios de los ingresos ocurren en los primeros 5 meses de vida y que el sexo masculino es el más frecuentemente afectado; ambos fueron observados en nuestro estudio, ya que el $64 \%$ correspondió al sexo masculino y el $96,4 \%$ de nuestros casos estuvo en el rango comprendido entre 21 días y 5 meses de $\operatorname{edad}^{19,20}$.

Como factores de riesgo, la prematuridad, la exposición al humo de tabaco, la ausencia de lactancia materna, el hacinamiento, entre otros, son considerados importantes en la severidad del cuadro y la presencia del VSR; sin embargo, en nuestro estudio otros factores fueron los asociados (ver cuadro No. 1).

De los tratamientos disponibles ninguno acorta el curso o acelera la resolución de los síntomas en las IRABs por VSR; sin embargo, la ribavirina inhalada puede reducir el tiempo de hospitalización y de ventilación mecánica, aunque no reduce la 


\section{Gráfico No. 1. Distribución Estacional}

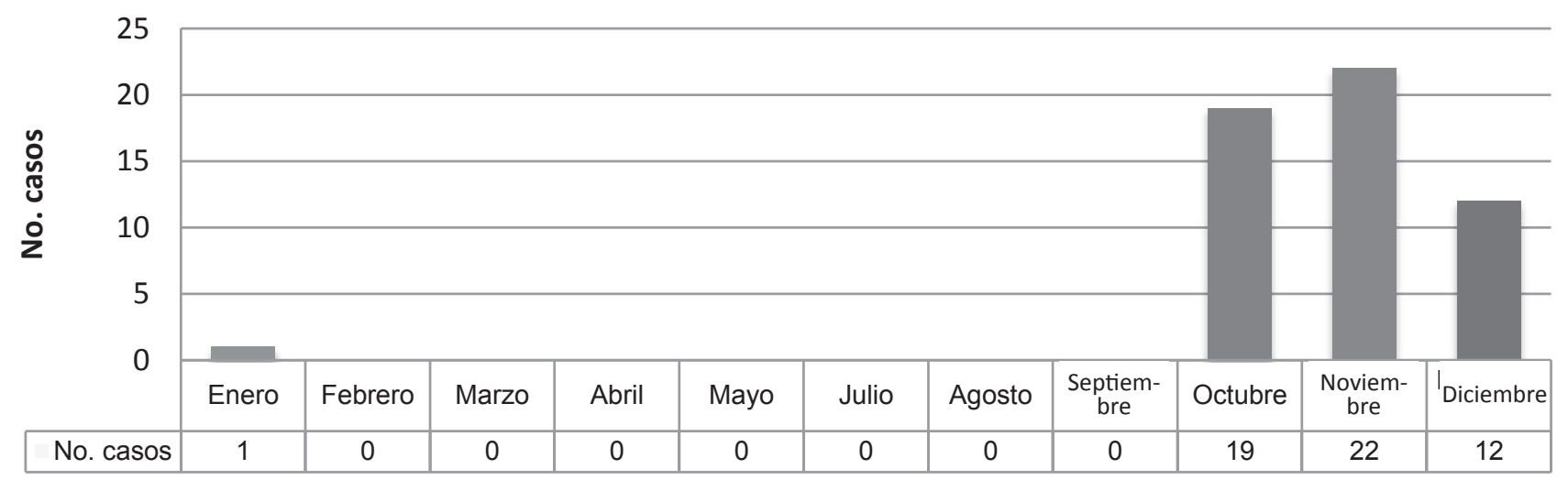

Cuadro 1. Factores de riesgo asociados a IRA por VRS

\begin{tabular}{lc}
\hline Factores de riesgo & No (\%) \\
\hline Tiene otros hermanos & $42(76)$ \\
Otro miembro de la familia con tos & $33(60)$ \\
Historia familiar de asma & $31(56)$ \\
Hermanos asisten a la escuela & $31(56)$ \\
Hacinamiento & $16(29)$ \\
Exposición a humo de tabaco & $15(22)$ \\
Prematuridad & $9(16)$ \\
No lactancia maternal & $8(15)$ \\
Desnutrición & $4(7)$ \\
Analfabetismo de los padres & $1(3,5)$ \\
\hline
\end{tabular}

Cuadro 2. Manifestaciones clínicas

\begin{tabular}{lc}
\hline Manifestaciones clínicas & No (\%) \\
\hline Tos & $53(98)$ \\
Dificultad respiratoria & $52(96)$ \\
Congestión nasal & $50(93)$ \\
Sibilancias & $47(87)$ \\
Anemia & $47(87)$ \\
Retracción subcostal & $46(85)$ \\
Retracción intercostal & $28(52)$ \\
Retracción supra-esternal & $22(41)$ \\
Crepitantes & $19(35)$ \\
Quejido & $15(28)$ \\
Cianosis & $9(7)$ \\
Rechazo alimentos & $7(13)$ \\
Fiebre & $6(11)$ \\
Desnutrición & $1(1,8)$ \\
\hline
\end{tabular}


mortalidad general. En cuanto a prevención, actualmente no hay vacunas disponibles, pero sí un único tratamiento preventivo, el palivizumab, recomendado en niños con comorbilidades que predisponen enfermedad grave y alto riesgo de mortalidad ${ }^{19.21}$.

Tos y dificultad respiratoria se presentaron en el 99\% de los casos, sibilancias en el $92 \%$ y dificultad respiratoria en el $88 \%$. El $80 \%$ presentó anemia, hallazgo no reportado en otros estudios (ver cuadro 2).

El $68 \%$ de los casos correspondió a bronquiolitis, $31 \%$ a neumonía intersticial y $1 \%$ atelectasia, hallazgos confirmados por radiografía de tórax.

No hubo mortalidad ni secuelas respiratorias, a pesar de que se conoce que esta enfermedad representa un riesgo de asma. Sería importante dar seguimiento a estos niños para conocer qué porcentaje de nuestros casos se asociarían a asma en la niñez, la adolescencia y la edad adulta temprana ${ }^{19}$.

\section{Aporte del estudio:}

En nuestro país se desconocía la carga que representaba el VRS como causa de IRABs. Conocer que VSR es el responsable del $48 \%$ de los casos de bronquiolitis y neumonía intersticial en niños menores de 7 meses y que tiene un comportamiento estacional, como sucede en todo el mundo, nos ayuda a mejorar el manejo, a controlar el uso de antimicrobianos y a fortalecer la necesidad de implementar programas de higiene de manos.

\section{Agradecimiento:}

A las doctoras Chabela Peña, Carmen Sara Mota y Rody Benítez, por su coloaboración en la recolección de datos.

\section{Referencias}

1. Callejón A, Hernández $\mathrm{O}$, Callejón G., Marrero C, Rodríguez E. Infección respiratoria por virus sincitial respiratorio. Bronquiolitis. BSCP Can 2004; 28(2 y 3): 249-263.

2. Ferres M, Perret P. proyecto Vigilancia de Virus Respiratorio 2005. Laboratorio de Infectología y Virología Molecular. Centro de Investigaciones Médicas. Escuela de Medicina. P. Universidad Católica de Chile.

3. Black CP. Systematic Review of the Biology Medical Management of Respiratory Sincytial Virus Infection. Respiratory Care. March 2003; 48(3):209-233

4. Aparato Respiratorio: Fisiología y Clínica Parte V: Enfermedades Respiratorias. Sección 2. Enfermedades infecciosas capítulo 28, infecciones de las vías aéreas. Pontificia Universidad Católica de Chile. Disponible en: http://www. virus.med.puc.cl/virusrespiratorios

5. Henrickson, K. Advances in the Laboratory Diagnosis of Viral Respiratory Disease. Pediatr Infect Dis J. 2004; 23(1s).

6. Cardozo Macedo S, Baptista Menezes A, Post P, Albernaz E, Knorst M. Respiratory Syncytial Virus Infection in Children under one year of age hospitalized for acute respiratory diseases in Pelotas, RS. J. Pneumologia. Jan./Feb. 2003; 29(1).

7. Bello O, Langenhin M, Pujadas M, Mateo S, Chiparelli H. Infecciones Graves por Virus respiratorio Sincitial en Lactantes menores de tres meses. Incidencia en pacientes sin factores de riesgo. Arch. Pediatr. 2001 nov; 72 (supl.1).

8. Comité Sociedad Argentina de Pediatría (SAP). Infección Respiratoria Aguda Baja. Arch Argent Pediatr. 2000; 98(3). 
9. Sato M, et al. Molecular Epidemiology of Respiratory Syncytial Virus Infection among Children with Acute Respiratory Symptoms in a Community over Three Seasons. J Clin Microbiol. 2005 jan; 43(1):36-40.

10. Murrara-Gutiérrez, A. y cols. Virus Sincicial Respiratorio en la Temporada Invernal 19992000 en la Ciudad de Monterrey, Nuevo León, México. Bol Med Hosp Infant Mex. 2001; 58(7):441-447.

11. Calegari $\mathrm{T}$ et al. Clinical-Epidemiological evaluation of Respiratory Syncytial Virus Infection in Children Attend in a Public Hospital in Midwestern Brazil. The Brazilian Journal of Infectious Diseases. 2005; 9(2):156-161.

12. Palomino MA, Larenas AJ, Moraga AG, Avendano C LF Severidad Clínica de la Infección Respiratoria Baja por Virus Sincitial por Grupos A y B. Rev. chil. pediatr. 2004 cot; 75 supl.1.

13. Howard MC, y cols. A Multicenter, Randomized, Controlled Trial of Dexametasone for Bronchiolitis. N, Engl J Med. 2007; 357(4):331-339.

14. Woensel, Aalderen, Kimpen. Clinical Review: Viral lower respiratory tract infection in infants and young children. BMJ. 20055 july; 327: 36-40.

15. Respiratory Syncytial Virus Infection in Ederly and High-Risk Adults. N Engl J Med. 2005; 352(17):1749-1759.
16. RSV Card Product Instructions, Alere BinaxNOW ${ }^{\circ}$ RSV Card. Disponible en: www. cliawaived.com

17. Nair H, Nokes DJ, Gessner BD, Dherani M, Madhi SA, Singleton RJ. Carga mundial de infecciones respiratorias agudas bajas por virus respiratorio sincicial en niños pequeños: una revisión sistemática y meta-análisis. Revisión Sistemática. Lancet. 2010.

18. Prevención de la Infección respiratoria aguda grave por virus sincitial respiratorio en pediatría: Anticuerpo monoclonal específico (Palivizumab). Lineamientos técnicos. Argentina. Ministerio de Salud. Presidencia de la Nación; 2014.

19. OMS | Centros para diseminar tecnología y salvar vidas. Disponible en: http://www.who. int/bulletin/volumes/93/5/15-020515/es/ visita el 3/6/18, 1:18 PM.

20. H Cody Meissner MD, Viral Bronchiolitis in Children. N Engl J Med. 2016 Jan; 374:6272. DOI: 10.1056/NEJMra1413456.

21. JM Galván et al. / Review of Non-bacterial Infections in Respiratory Medicine: Viral Pneumonia . Arch Bronconeumol. 2015;51(11):590-597. Disponible en: http:// www.elsevier.es, day 06/03/2018. 